\title{
RESEARCH OF DECISION SUPPORT SYSTEM (DSS) FOR GREENHOUSE BASED ON DATA MINING
}

\author{
Cheng Wang $^{1}$, Lili Wang ${ }^{2, *}$, Ping Dong ${ }^{2}$, Xiaojun Qiao ${ }^{1}$ \\ ${ }^{1}$ National Engineering Research Center for Information Technology in Agriculture, Beijing, \\ China \\ ${ }^{2}$ Information engineering institute University of Science and Technology Beijing, Beijing, \\ China \\ * Corresponding author, Address: Shu Guang Hua Yuan Middle Road 11\#, Haidian district, \\ Beijing, 100097, P. R China, Tel: +86-010-51503409, Fax: +86-010-51503349, Email: \\ lippery@sina.com.cn
}

Abstract: Most of expert knowledge in agriculture is descriptive and experiential, so it is difficult to describe in mathematics and build decision support system (DSS) for greenhouse. Therefore, the decision support system (DSS) for greenhouse constructed of data warehouse and date mining technology was introduced in this paper. In the system, data warehouse was founded to memory diversified date, the using of on-line analytical processing and date mining enriches knowledge base with new agriculture information. Implementation of system adopted SQL Server analysis, as a result, tightness coupling of data warehouse, date mining and application, improved efficiency of date mining. Combined data warehouse with on-line analytical processing and date mining to construct a novel DSS.

Keywords: Data warehouse, Date mining, OLAP, Decision support system (DSS)

Wang, C., Wang, L., Dong, P. and Qiao, X., 2008, in IFIP International Federation for Information Processing, Volume 259; Computer and Computing Technologies in Agriculture, Vol. 2; Daoliang Li; (Boston: Springer), pp. 1071-1076. 


\section{INTRODUCTION}

Dates in agriculture have the follow characteristics: large quantity, multidimension, no integrity, incertitude and so on. How to effectively find the interrelationship of dates effects agricultural development, economic benefit, social benefit. Using decision support system (DSS) can obtain benefits (Zhao et al., 2006).

Now, expert knowledge base in most DSS is established on a domain, but acquisition domain expert knowledge is a complex process, describe in mathematics is difficult, along with quantity of data augment, the conventional DSS shows its inherent shortage. Development of data warehouse, on-line analytical processing (OLAP) and date mining improve DSS, and have breakthrough (Helen, 2001).

Data warehouse is dates gather aimed at thematic, co positive, steady, and multi-time. It contains basic data, historical date, synthetic data, and source date (Michael, 2001).These dates restructure according to decision convenient for user to extract each wished date and information. On-line analytical processing (OLAP) offers multi-dimension analysis method: slice up, slice block, rotation, etc. it also convenient for user to extract the needed date and information in differ point of view (Dorian, 1999). Based on OLAP and Data warehouse, Date mining can using correlation analysis, similitude search, trend analysis and forecast, pattern analysis to acquire new knowledge, find conclude reasoning and potential mode, help making correct decision. Above three Technologies have internal relation and complementarily, combining them can construct a new DSS (Cody, 2002).

\section{THE GENERAL DESIGN}

In this system, data warehouse memory dates from idiosyncratic information source, and that, these information source itself is a gigantic data warehouse, OLAP dedicated to date analysis, Date mining apply itself to acquire new knowledge, when design and construct DSS, apply above technology, can apply improve processing capacity.

Architectural structure of DSS as graph 1, data warehouse can realize storage of decision-making date, sampled data from source date, then cleaning, integrating, transferring, offering date view, and then these dates have good quality. OLAP can realize multi-dimension date analysis via building multi-dimension data model in multi-view. date mining automatically dig out the hidden mode and information, forecast the prospective trend, direction OLAP, expert system (ES) can utilize knowledge reasoning qualitative analysis, we call it competitive synthetic 


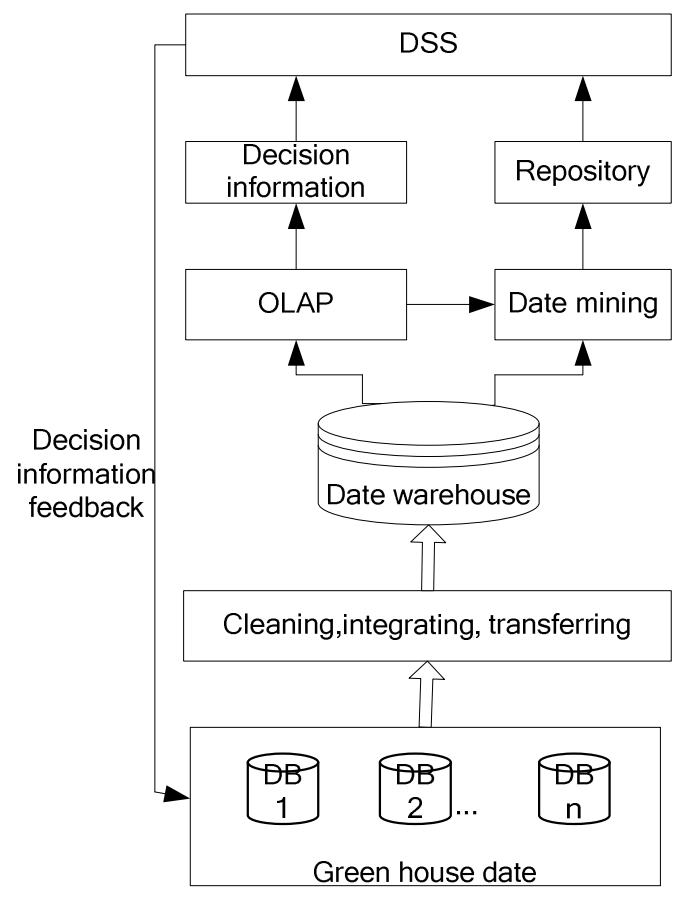

Fig. 1. System whole structure chart

decision support system, complementation, exert each other superiority, realize effective aid decision making (Inmon, 2002).

\section{DATA WAREHOUSE MODEL DESIGN}

First, Extraction related data environmental monitoring, building data warehouse. Then, On base of date warehouse, using date mining technology, dig out the hidden information, relationship of environmental element and restrict factor, improve utilance of monitoring dates, propose pertinence resolve means, advanced environment administrative department decisionmaking ability.

According as analysis, determinate issue include time, outside climate, environmental parameters, crop growth state, plant diseases and insect pests, invested funds. In this paper, we choose star model, for this model has the merit: convenience to build model, easy to understand, support multidemotion date analysis. Structure is Fig. 2. 


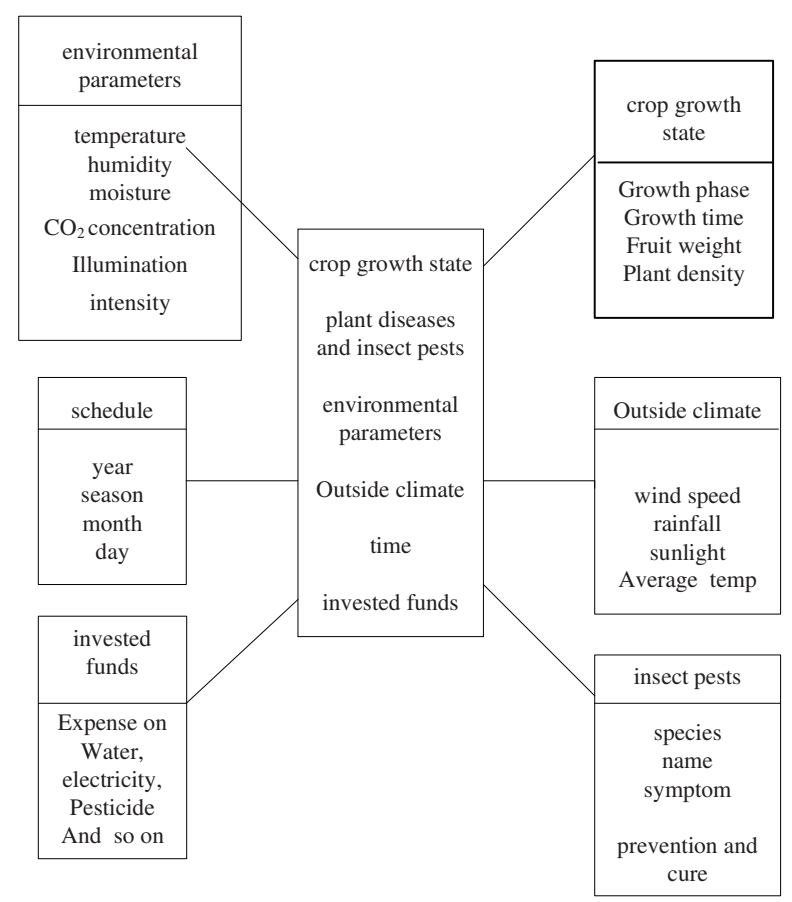

Fig. 2. Star model of data warehouse

\section{BUILD GREENHOUSE DSS}

\subsection{Build OLPA module}

To express the data in the data-base which using Service module of Microsoft OLAP Analysis Services, and basing on the fact dimension table. The method is slice up, slice block, rotation, to analyze relate dates in different view. OLAP model can complete analyzing environmental parameters, crop growth state, plant diseases and insect pests, then give out result. The result is easy to understand in mode of report forms, chart etc. PivotTables Service provide interface, adopt XDM sentence to perform multi-dimension date set search.

\subsection{Build date mining module}

Using multi-dimension date set build in OLAP to build date mining model. API -OLEDB for Data Mining is one of Analysis Services, it is a 
program interface for date mining application program, by means of API, using different kind of arithmetic to complete mining task.

\subsubsection{Trend analysis and forecast module}

For greenhouse, under the condition of forecast precision, can using prior three days dates to forecast the fourth day, that is to say, found a four section window. When greenhouse sensor fault or don't send dates, manager can take correspond means to maintain environmental parameters in perfect.

\subsubsection{Similitude search module}

In system, familiar module in different environmental parameters is similitude, find out comparability of modules. Similar module is temperature and illumination, air humidity and soil moisture and so on.

\subsubsection{Associate rule analysis module}

In DSS, based on plant diseases and insect pest's experience, using Apriori arithmetic, discover the associate rule of plant diseases and insect pest and environmental parameters.

\subsection{Program UI}

Visual C++ is a very good program tools, applications have friendly user interface, user can clean, integrity, transfer dates to date warehouse, also can build date warehouse, search date, give out result, etc. Build interested date mining module is allowed.

\subsection{System architecture}

This DSS consist of client, application server, database server. In client, using Visual C++ programmed applied UI, OLAP and date mining run in application server, bottom date warehouse build in database server. Client is used to report result for user, application serve handing applied logic, acquire date from database server when necessary, and deliver date to client. Database server automatic update maintenance date warehouse. 


\section{SUMMARIZE}

In this paper, we attempt to apply date mining and data warehouse technology in DSS of greenhouse, considering of greenhouse's characteristic, preliminary design the frame of data warehouse, on the base of date warehouse, we perform date mining, in order to offer useful content such as environmental parameters,, it's relation, plant diseases and insect pests for agriculture expert. In future, along with the development of data warehouse, OLAP and Date mining, DSS for greenhouse will have a perfect futurity.

\section{ACKNOWLEDGEMENTS}

This research was supported by the National High Technology Research and Development Program of China (863 Program, Grant 2006AA10A311) and National High Technology Research and Development Program of China (863 Program, Grant 2006AA10Z253).

\section{REFERENCES}

Helen Hasan, Peter Hyland. Using OLAP and multidimensional data for decision making. IT Professional, Vol. 3, No. 5, 2001, pp. 44-50

Michael Blaha. Data warehouses and decision support systems. M Computer, Vol. 34, No. 12, 2001, pp. 38-39

Dorian Pyle. Data Preparation for Data Mining. Morgan Kaufmann, 1999

Inmon W H. Building the Data Warehouse (Third Edition). John Wiley \& Sons, 2002, pp. 20-53

Y W F, Kerulen J T, Krishna V, Spangler W S. The Integration of Business Intelligence and Knowledge Management. IBM Systems Journal, Vol. 41, No. 4, 2002, pp. 697-714

Zhao Yu, Q I Guoqiang. Application of data warehouse in decision support system of rice cultivation management [J]. Journal of Northeast Agricultural University, Vol. 37, No. 4, 2006, pp. 557-562 